\title{
HALO ESTIMATES AND SIMULATIONS FOR LINEAR COLLIDERS
}

\author{
H. Burkhardt, L. Neukermans, A. Latina, D. Schulte, CERN, Geneva, Switzerland; \\ I. Agapov*, G. A. Blair (Royal Holloway, University of London; Surrey) \\ F. Jackson (STFC/DL/ASTeC, Daresbury, Warrington, Cheshire)
}

\section{Abstract}

Halo simulations and estimates are important for the design of future linear accelerators. We describe the main processes with analytic estimates and present our generic simulations in application to the ILC.

\section{INTRODUCTION}

Halo particles contribute very little to the luminosity but may instead be a major source of background and radiation [1]. Even if most of the halo will be stopped by collimators, the secondary muon background may still be significant [2, 3].

We study halo production with detailed simulations, to accompany the design studies for future linear colliders such that any performance limitations due to halo and tails can be minimised [4].

We calculate and simulate particle scattering and radiation processes. Core particles can significantly increase in amplitude and become halo particles by the following processes :

- Beam Gas elastic scattering, multiple scattering

- Beam Gas inelastic scattering, Bremsstrahlung

- Scattering off thermal photons

- Intrabeam scattering

- Synchrotron radiation

Experimental evidence for the importance of particle scattering processes in the production of halo particles was in observed storage rings [5].

Intrabeam scattering is important at low energies and in particular in the damping ring. The simulation of this processes is currently outside the scope of this study.

In addition to particle scattering processes, optics related effects like mismatch, coupling, dispersion and nonlinearities can enhance the beam halo. This is studied by combining the halo generation with detailed tracking programs and also allows to study the effect of wake fields, alignment and ground motion on the core and halo of the beam. We currently provide interfaces between our halo generation by particle processes and the optics tracking programs like PLACET [6] and MERLIN [7]. Synchrotron radiation is included in the tracking programs and has recently been further optimised [8].

The Monte Carlo halo generator code for the particle scattering processes is provided as program package HTGEN [9]. The package includes documentation, installa- tion instructions, standalone test procedures for each process as well as interface routines for the PLACET and MERLIN programs.

In the following, we will look closer into the use and application of the halo generation package. For the illustration and estimates, we will concentrate here on the LINAC and BDS sections of the ILC and refer to [4] for CLIC.

\section{MAIN PROCESSES}

\section{Elastic scattering}

In the elastic process of Mott scattering, the incident beam particle is deflected by the Coulomb potential of the particles in the residual gas. Elastic scattering changes the direction of the beam particle while its energy is not affected. Elastic scattering can lead to large betatron amplitudes and loss of particles at collimators or any other aperture restriction.

The angular distribution of the scattered electron is given by the differential Mott cross section [10]

$$
\frac{\mathrm{d} \sigma}{\mathrm{d} \Omega}=\left[\frac{Z r_{e}}{2 \gamma \beta^{2}}\right]^{2} \frac{1-\beta^{2} \sin ^{2} \frac{\vartheta}{2}}{\sin ^{4} \frac{\vartheta}{2}},
$$

where $Z$ is the charge of the nucleus, $r_{e}$ the classical electron radius and $\gamma$ the Lorentz factor $E / m c^{2}$ of the electron. Note that $\beta$ is here the velocity in units of the speed of light. For a simple estimate, we use $\beta=1, \sin (\vartheta / 2) \approx \vartheta / 2$ and see that the angular distribution is dominated by the Coulomb term $16 / \theta^{4}$

The total cross section is obtained by integration over the solid angle. Relevant for halo production are scattering angles which exceed the beam divergence, or roughly

$$
\theta_{\min }=\sqrt{\epsilon / \beta_{y}}=\sqrt{\epsilon_{N} / \gamma \beta_{y}}
$$

where $\epsilon_{N}=\gamma \epsilon$ is the normalized vertical emittance and $\beta_{y}$ the local vertical beta function. Using the same approximations as above, we get as a simple estimate for the integrated elastic cross section as a function of the minimum scattering angle

$$
\sigma_{\mathrm{el}}=\frac{4 \pi Z^{2} r_{e}^{2}}{\gamma^{2} \theta_{\min }^{2}}
$$

Using Eq. 2 we can rewrite the cross section in terms of the normalized emittances [11] as

$$
\sigma_{\mathrm{el}}=\frac{4 \pi Z^{2} r_{e}^{2} \beta_{y}}{\epsilon_{N} \gamma}
$$

* now at CERN

05 Beam Dynamics and Electromagnetic Fields
D03 High Intensity - Incoherent Instabilities, Space Charge, Halos, Cooling 
At constant normalized emittance, the elastic cross section scales inversely with energy.

\section{Inelastic scattering}

At high energy, the dominating process relevant for energy loss or inelastic scattering is Bremsstrahlung in which the incident electron interacts with the field of the residual gas nucleus and radiates photons. The energy spectrum is rather broad and can be approximately written as

$$
\frac{d \sigma}{d k}=\frac{A}{N_{A} X_{0}} \frac{1}{k}\left(\frac{4}{3}-\frac{4}{3} k+k^{2}\right),
$$

where $k$ is the photon energy in units of the beam energy, $N_{A}$ the Avogadro constant, $X_{0}$ and $A$ are the the radiation length and the mass of the material. Integration over $k$ (from $k=k_{\min }$ to $k=1$ ) yields

$$
\sigma_{\text {in }}=\frac{A}{N_{A} X_{0}}\left(-\frac{4}{3} \log k_{\min }-\frac{5}{6}+\frac{4}{3} k_{\min }-\frac{k_{\min }^{2}}{2}\right) .
$$

The cross section does not vary with energy at fixed $k_{\min }$. For $k=k_{\min }=0.01$ the inelastic cross section is 0.375 Barn per Helium Atom and 6.510 Barn for $N^{2}$ for the sum of the two nuclei. The angular cross section is given by

$$
f(\theta) d \theta \propto \frac{\theta d \theta}{\left(\theta^{2}+\gamma^{-2}\right)^{2}} .
$$

\section{Scattering off thermal photons}

Even for a perfect vacuum, the beam pipe will be "filled" with thermal photons. The photon density scales with the absolute temperature $T$ as $T^{3}$ and reaches $\rho_{\gamma}=5.32 \times$ $10^{14} \mathrm{~m}^{-3}$ at room temperature. The cross section for scattering off thermal photons is close to the Thomson cross section $\sigma_{T}=\frac{8 \pi}{3} r_{e}^{2}=0.665$ Barn. From the product of cross section and photon density we obtain a scattering probability of $3.5 \times 10^{-14} / \mathrm{m}$ and electron at room temperature. The mean energy loss scales about linearly with the energy of the electron and reaches $5.3 \%$ at $250 \mathrm{GeV}$ and $75 \%$ of the electrons will loose at least $1 \%$ of their energy (and 18\% more than 10\%) in this scattering. Details of the simulation of this process are described in [12].

\section{DISCUSSION FOR THE ILC}

We use the lattice and nominal beam parameters of the ILC as described in the latest (March 2007) baseline configuration document [13].

The beam gas estimates in the BDS section were performed for nitrogen at room temperature at a pressure of 50 nTorr. For the LINAC section we use Helium at 2 Kelvin and 10 nTorr.

The results of simple analytic estimates for elastic scattering are presented in Table 1. The scattering probabilities at the end of the LINAC and in the BDS per unit length are similar. The probability for elastic scattering at the beginning of the LINAC is about 50 times higher. By integration
Table 1: Analytical estimates for elastic beam-gas scattering with scattering angles above $1 \sigma$ of the beam divergence calculated for a constant normalized emittance of $20 \mathrm{~nm}$ at $\beta_{y}=100 \mathrm{~m} . \quad \rho$ is the density of He atoms or nitrogen molecules per $m^{3}$ and $P$ the scattering probability per electron and meter.

\begin{tabular}{cccccc}
\hline Location & $\mathrm{E}$ & $\mathrm{Gas}$ & $\rho$ & $\sigma_{\text {el }}$ & $P$ \\
& $\mathrm{GeV}$ & & $m^{-3}$ & Barn & $m^{-1}$ \\
LINAC & 5 & $\mathrm{He}$ & $4.8 \times 10^{16}$ & $2.0 \times 10^{6}$ & $9.9 \times 10^{-6}$ \\
LINAC & 250 & $\mathrm{He}$ & $4.8 \times 10^{16}$ & $3.8 \times 10^{4}$ & $1.8 \times 10^{-7}$ \\
BDS & 250 & $\mathrm{~N}_{2}$ & $1.6 \times 10^{15}$ & $4.6 \times 10^{5}$ & $1.5 \times 10^{-7}$ \\
\hline
\end{tabular}

over the LINAC we find that an electron has a probability of about $9 \times 10^{-3}$ to undergo elastic scattering with an angle of at least the beam divergence. Only a fraction of these will hit spoilers or the beam pipe.

To get a very rough analytic estimate of the probability for particle loss by elastic scattering we have also calculated the probabilities for scattering at angles exceeding 30 times the vertical beam divergence. The probability integrated over the LINAC is about $10^{-5}$. Integrated over the BDS we get a probability of about $5 \times 10^{-7}$.
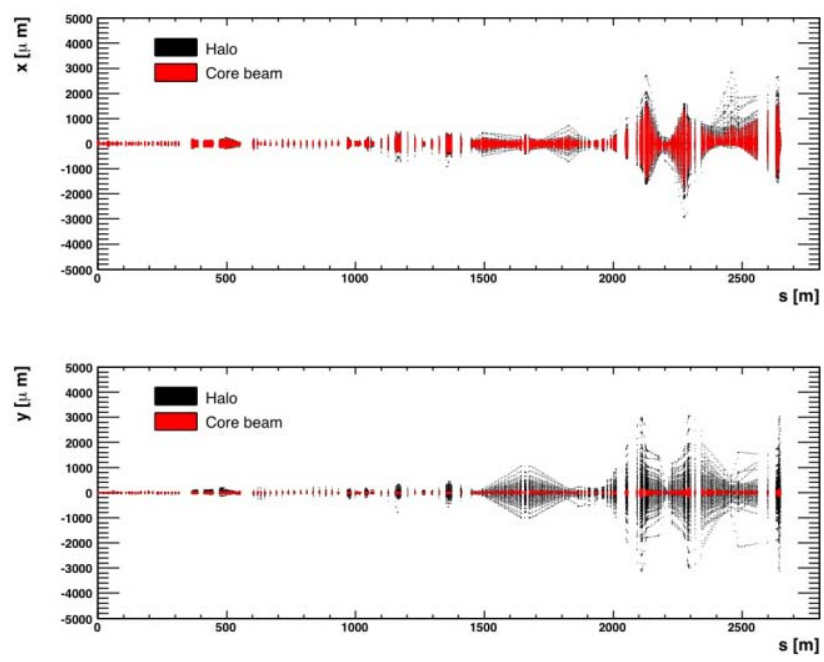

Figure 1: Tracking example for the ILC. Horizontal (top) and vertical (bott) beam positions as function of the longitudinal coordinate $s$ in the BDS. Halo particles are shown in black and core beam particles in red.

The probability for inelastic scattering with a fractional energy loss $k_{\min }>0.01$ is small, $1.8 \times 10^{-12} / \mathrm{m}$ in the LINAC and rather similar, $1.0 \times 10^{-12} \mathrm{~m}$ in the BDS. Summing up LINAC and BDS, we get a probability for inelastic scattering of $2.3 \times 10^{-8}$. The probability of thermal scattering is still much smaller, about $9 \times 10^{-11}$ for the BDS and completely negligible for the (cold) LINAC.

More detailed information is obtained from the simulation. Figs 1 and 2 illustrate the results of our tracking studies performed for the ILC using PLACET. In the simulation, the beam gas pressure and apertures can be separately 

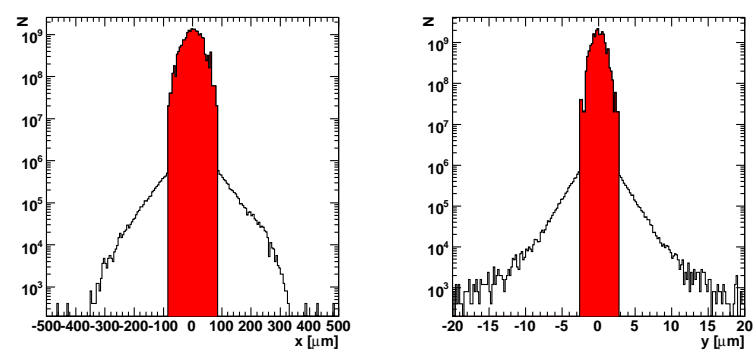

Figure 2: Transverse beam profiles at the BDS entrance.

specified for each element. Variations of optical parameters are fully taken into account. Particles hitting the beam pipe are normally considered to be lost. For particles hitting the spoiler instead, we implemented a simulation of multiple scattering and energy loss by ionization and checked this by comparison with GEANT4 [14].

The simulations described here were performed for an ideal machine without errors and positioning tolerances. We have started to assign random position and gradient errors in our simulations and find that this results into enhanced tail distributions.

In the idealized simulation without errors, we find that the beam-gas scattering from the LINAC and BDS combined results in a fraction of $10^{-4}$ of the particles impacting on the spoilers. For the nominal intensity of $2 \times 10^{10}$ particles per bunch and 2820 bunches, we expect that $6 \times 10^{9}$ particles hit the spoilers at each train crossing. Based on earlier estimates [3], this will result in roughly $10^{5} \mathrm{sec}$ ondary muons.

\section{SUMMARY AND OUTLOOK}

We provide a generic package HTGEN and specific interfaces for halo tail simulations in linear colliders. We have used the package in CLIC and ILC studies. We also discuss approximate analytic expressions, and described these here together with simulations performed for the LINAC and BDS sections of the ILC.

We have started to compare our code with other simulation programs, and in particular BDSIM [15] and GEANT4 [14]. We find that our code and these programs are rather complementary. BDSIM/GEANT4 allow for simulations of many processes and they are well adapted to simulate cascades and multiple scattering in dense materials. Our HTGEN program is instead well adapted to simulate relatively rare single scattering progress.

We plan to combine HTGEN and BDSIM and to investigate benchmarking with halo measurements in CTF3 and ATF.

\section{ACKNOWLEDGEMENT}

This work is supported by the Commission of the European Communities under the $6^{\text {th }}$ Framework Programme 05 Beam Dynamics and Electromagnetic Fields

D03 High Intensity - Incoherent Instabilities, Space Charge, Halos, Cooling
"Structuring the European Research Area", contract number RIDS-011899.

\section{REFERENCES}

[1] H. Burkhardt, "General machine background in future $\mathrm{e}^{+} \mathrm{e}^{-}$colliders", Proc. of the Internat. Workshop on Linear Colliders, LCWS99, 1999 Sitges/Barcelona and CERN-SL-99-057-AP.

[2] L. P. Keller, "Muon background in a $1-\mathrm{TeV}$ linear collider", Contributed to 5th International Workshop on Next-Generation Linear Colliders, Stanford, CA, 13-21 Oct 1993, SLAC-PUB-6385.

[3] G. A. Blair, H. Burkhardt, and H. J. Schreiber, "Background simulation for the CLIC beam delivery system with Geant", Proceedings of EPAC 2002, Paris, France, pp. 449-451.

[4] H. Burkhardt, L. Neukermans, and J. R. Lopez, "Halo and tail generation studies for linear colliders", Proc. EPAC 2006, CLIC-NOTE-668 and EUROTeV-Report-2006-028.

[5] H. Burkhardt, I. Reichel, and G. Roy, "Transverse beam tails due to inelastic scattering", PRSTAB 3 (2000) 091001.

[6] A. Latina, H. Burkhardt, P. Eliasson, L. Neukermans, J. R. Lopez, G. Rumolo, D. Schulte, and R. Tomas, "Recent improvements of PLACET", Proc. EPAC 2006 and EUROTEV-REPORT-2006-030.

[7] D. Krucker, F. Poirier, and N. Walker, "An ILC main linac simulation package based on Merlin", Proc. EPAC 2006 and EUROTEV-REPORT-2006-071.

[8] H. Burkhardt, "Monte Carlo Generation of the Energy Spectrum of Synchrotron Radiation", CLIC-Note 2007-709 and EUROTeV Report 2007-018.

[9] H. Burkhardt and L. Neukermans, "Halo and Tail generator package htgen", http://hbu.home.cern.ch/hbu/HTGEN.html.

[10] W. Williams, Nuclear and Particle Physics. Oxford University Press, 1991.

[11] R. Brinkmann. Presentation at BDIR-2000 in Daresbury .

[12] H. Burkhardt, "Monte Carlo Simulation of Beam Particles and Thermal Photons", SL Note 93-73 (OP), CERN, July, 1993.

[13] "The International Linear ColliderGlobal Design Effort Baseline Configuration Document", available from http://www.linearcollider.org/wiki/doku.php.

[14] S. Agostinelli et al., "GEANT4: A simulation toolkit", Nucl. Instrum. Meth. A506 (2003) 250-303.

[15] I. Agapov, G. A. Blair, J. Carter, and O. Dadoun, "BDSIM: Beamline simulation toolkit based on GEANT4", Proc. EPAC 2006. 